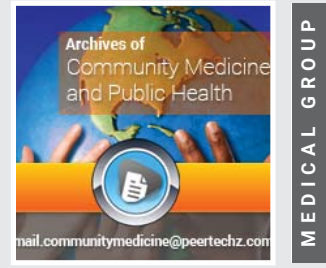

\title{
Social isolation, loneliness and health in the time of COVID
}

\section{Thomas L Campbell*}

Professor, Department of Family Medicine, University of Rochester School of Medicine and Dentistry,

New York, USA
Received: 13 June, 2020

Accepted: 19 June, 2020

Published: 20 June, 2020

*Corresponding author: Thomas L Campbell MD, Professor, Department of Family Medicine, University of Rochester School of Medicine and Dentistry, New York, USA, Tel: 585-746-9715;

E-mail:Tom_Campbell@urmc.rochester.edu

https://www.peertechz.com

Check for updates
Humans are social beings. We rely on others for our survival and well-being. Lack of social relationships have an enormous impact on our physical and emotional health and are a stronger risk factor for early mortality than most traditional health risk factors [1]. Our social connections have been threatened by the COVID-19 pandemic

Social isolation is an objective state of having minimal social contact with others. Loneliness is a subjective state of lacking a desired level of connection with others. They are related but have little overlap or correlation with each other [2]. Individuals may be socially isolated, but not distressed or feeling lonely. Others may feel lonely among many social contacts. One quarter of older adults report social isolation and nearly 50\% experience loneliness [3].

Both social isolation and loneliness have significant physical and mental health effects, especially at older ages. A recent meta-analytic review of 70 prospective studies involving more than 3 million adults found that those reporting loneliness had a $26 \%$ increased likelihood of dying prematurely and those who were socially isolated had a $29 \%$ risk of early death [4]. The health effects of social isolation and loneliness appear to be synergistic, such that those who experience both are at particularly high risk of early death [5]. Besides early mortality, loneliness and social isolation have also been associated with a wide range of health conditions, including depression, suicide, dementia, hypertension, cardiac disease and stroke [6]. Elevated blood pressure, abnormal HPA response, decreased immunity and chronic inflammation have all been associated with loneliness and social isolation

As a result of the COVID-19, the public has been asked to practice social distancing and social isolation. These are unfortunate and inaccurate terms. To prevent transmission of the virus, individuals must be physically distant, but not necessarily socially distant or isolated. The key to healthy physical distancing and isolation is to remain socially connected which can be particularly challenging for older adults.

Clinicians are in a unique position to address the problem of social isolation and loneliness of the elderly because even the most socially isolated have regular contact with their medical providers. During the pandemic most non-urgent clinical visits have been replaced by tele-visits, often for the management of chronic disease. These visits can be used to assist older patients suffering from loneliness or social isolation.

Prior to conducting a tele-visit, the clinician should review the patient's chart to see what risk factors the patient may have for social isolation and loneliness. Mental health problems, functional disabilities, sensory impairments, especially hearing loss, and recent divorce or widowhood have all be identified as risk factors [3]. When appropriate, depression screening should considered before the visit. Although there are no clinically validated screening instruments, the Berkman-Syme (for social isolation) and the UCLA Loneliness scale (for loneliness) have been recommended for clinical use due to their brevity and validity [3].

When possible, patients should be encouraged to do televisits by video rather than telephone visits. More information can be obtained by observing the patient [7]. A patient's appearance, dress and affect may give important clues as to how they are coping with physical distancing. Unfortunately, there remains a significant "digital divide" for older adults which is even greater for those who are rural, disadvantaged, or disabled. Many older patients do not have a smart phone or computer with internet access. Medical assistants or secretaries can work with patients to help set up these appointments. The inability to connect by video with family or friends may be a sign of social isolation.

Begin virtual visits with an open ended question such as "How are you doing?" or "How are you coping"? Inquire who 
is at home with them, and how they are staying connected with family and friends. Stress the importance of staying socially connected while remaining physically isolated. For patients who live alone, further investigation of their social network and support system is important. How are they staying connected to their families? Who is delivering food and medication? Are they lonely?

Much has been written about how to cope with physical isolation, but some specific recommendations can be given to older patients to increase their social connections. Scheduling regular contact with family and friends by telephone or preferably by video can help reduce social isolation. AARP has provided advice on how to cope with social isolation during COVID epidemic as well as instructions for older adults on how to set up a FaceTime call [8].

There are no established guidelines or proven interventions to help patients who are experiencing social isolation or loneliness. For busy clinicians, the most practical "social prescription" is to refer the patient to a social worker or other health care professional within the practice or community to do a more detailed evaluation of the patient's needs. Other social prescriptions may include direct referrals to community agencies that help directly with social isolation and loneliness or address specific needs such as Meals on Wheels. Most communities have developed websites devoted to resources for the COVID pandemic.

Patients suffering from a mental health disorder, most commonly anxiety or depression, should be referred for mental health services, preferably to an on-site counselor who can do further assessment and virtual counseling for these patients. On-line cognitive-behavioral therapy has been demonstrated to be effective in treating loneliness and depression [9]. Patients with depression should always be assessed for suicidality and managed accordingly.

The National Academy of Science, Engineering and Medicine recently published a comprehensive report on social isolation and loneliness in older adults with an emphasis on opportunities for health care professionals [3]. They provide a detail list of recommendations for further research, expanded education of health care workers, and better integration of clinical practice and social organizations to address these problems. We are in the midst of a deadly pandemic that threatens older adults not just with a lethal infection, but by worsening social isolation and loneliness and their resulting health problems. Clinicians on the front lines can address and mitigate the effects of social isolation and loneliness in their older patients.

\section{References}

1. House JS, Landis KR, Umberson D (1988) Social relationships and health. Science 241: 540-545. Link: https://bit.ly/2Ycyuon

2. Coyle CE, Dugan E (2012) Social isolation, loneliness and health among older adults. J Aging Health 24: 1346-1363. Link: https://bit.ly/3fFo8Dq

3. National Academies of Sciences Engineering, and Medicine 2020. Social isolation and loneliness in older adults: Opportunities for the Health Care System. The National Academies Press.

4. Holt-Lunstad J, Smith TB, Baker M, Harris T, Stephenson D (2015) Loneliness and social isolation as risk factors for mortality: a meta-analytic review. Perspect Psychol Sci 10: 227-237. Link: https://bit.ly/3djw5wd

5. Beller J, Wagner A (2018) Loneliness, social isolation, their synergistic interaction and mortality. Health Psychol 37: 808-813. Link: https://bit.ly/2CnDrSP

6. Ong $A D$, Uchino $B N$, Wethington $E$ (2016) Loneliness and health in older adults: A mini-review and synthesis. Gerontology 62: 443-449. Link: https://bit.ly/3hJzwjd

7. Donaghy E, Atherton H, Hammersley V, McNeilly H, Bikker A, et al. (2019) Acceptability, benefits, and challenges of video consulting: a qualitative study in primary care. Br J Gen Pract 69: e586-e594. Link: https://bit.ly/2YhuPpr

8. AARP (2020) How to make a FaceTime call on your Iphone, Ipad, Ipad touch Mac. Link: https://bit.ly/2YPt69J

9. Käll A, Jägholm S, Hesser H, Andersson F, Mathaldi A, et al. (2020) Internetbased cognitive behavior therapy for loneliness: a pilot randomized controlled trial. Behavior Therapy 51: 54-68. Link: https://bit.ly/3fBDE36

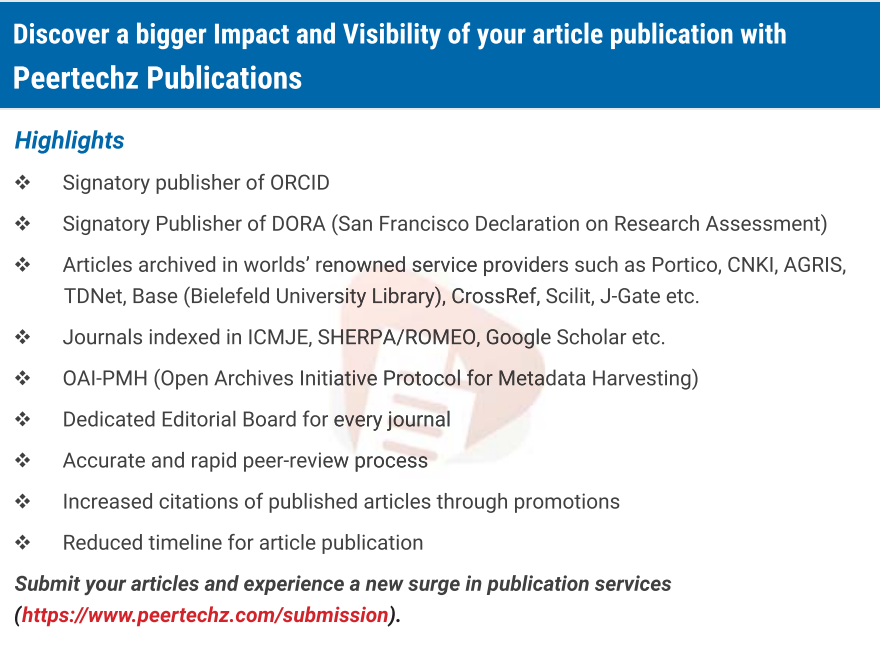

Copyright: @ 2020 Campbell TL. This is an open-access article distributed under the terms of the Creative Commons Attribution License, which permits unrestricted use distribution, and reproduction in any medium, provided the original author and source are credited. 